\title{
Vertebral Arteriovenous Fistula
} Associated with Neurofibromatosis Type I
Misdiagnosed as a Giant Aneurysm*

\author{
G. BENNDORF, U. ASSMANN***, A. BENDER**, T. N. LEHMANN*, W. R. LANKSCH* \\ Department of Radiology, and * Neurosurgery, Virchow Hospital, Charite, HU Berlin \\ ** Radiologische Diagnostik Kapweg, Berlin \\ *** Department of Neurosurgery, Frankfurt-Hoechst
}

Key words: AVF, neurofibromatosis, aneurysm

\section{Summary}

A 59-year-old man with neurofibromatosis type 1 (NF1) presented with bruits and neck pain due to a space occupying lesion in the right neck tissue. Digital subtraction angiography $(D S A)$ showed an arteriovenous fistula $(A V F)$ of the right extracranial vertebral artery (VA) with a giant venous pouch and an intracranial berry aneurysm of the right middle cerebral artery (MCA). First, the MCA aneurysm was surgically clipped, then the patient was treated by embolisation with coils. The coils were placed transarterially from the left VA resulting in a partial thrombosis of the venous pouch. Complete closure was achieved secondarily by retrograde transvenous catheterization. Etiology and treatment modalities are discussed.

\section{Introduction}

In NF1, vascular lesions presenting as stenoses, occlusions and aneurysmal or fistulous malformations are well known. They often occur in branches of the abdominal aorta, thus more often in the renal than in the cerebral arteries ${ }^{1,2,3}$.

The most common manifestation is stenosis of the renal artery, causing renovascular hypertension ${ }^{2,4,5}$. Cases of occlusion of the internal carotid and/or middle cerebral artery followed by development of collateral vessels with a moya-moya-like appearance are not uncommon ${ }^{3,6}$. Association with arteriovenous anomalies of the central and peripheral nervous system is rare, but these malformations can become fairly large, extending mainly in a craniocaudal direction ${ }^{7}$.

In the present paper, we report on a patient with NF1 and an arteriovenous fistula of the proximal vertebral artery that drained into a giant venous pouch causing bruits and mass effect.

Today, as described by different authors ${ }^{1,2,8,9}$, endovascular occlusion by balloons, coils or NBCA represents the most feasible method for effective treatment of these lesions. We were able to close such a fistula by occluding the venous outflow using a transfemoral venous approach.

\section{Case history}

A 59-year-old patient with known NF1 suffering from arterial hypertension and coronary heart disease presented with a right-sided soft mass in the neck tissue, which had caused intermittent pain for six months. The patient also had a history of polycystic kidney disease, chronic kidney insufficiency and multiple hepatic cysts.

There was no history of trauma, bleeding or neurological symptoms. A general physical ex-

* The case reported here was published as "Giant aneurysm" in Neuroradiology 40: 245-248, 1998 without the authors' (G.B., U.A., A.B., T.L., W.L.) consent. 
amination showed multiple neurofibromas and "café-au-lait" spots on the neck and trunk. Inspection of the head and neck showed a large mass in the right sternoclavicular angle with a palpable bruit. All peripheral pulses were normal, a neurological examination detected no abnormalities. A systolic-diastolic machinery murmur with the maximum over the pulmonary artery and conduction to the right carotid artery was discovered by heart auscultation. An ultrasound of the right sternoclavicular angle revealed a fluid-filled large cavity, measuring 51 x $88 \mathrm{~mm}$.

Initial DSA (figure 1) showed a large saccular lesion in the right neck, consisting of two connected venous pouches. The smaller pouch (ca. $20 \times 25 \mathrm{~mm}$ ) was filled by the short, caudally distorted segment of the proximal right VA, whereas the larger one (ca. $60 \times 100 \mathrm{~mm}$ ) was filled mainly by the distal right VA in a retrograde fashion.

The left VA and basilar artery (BA) were of a large diameter with multiple irregularities of the wall but without severe stenoses. Even in very late phases of the arteriogram, it was impossible to recognize a venous exit. A vertebral arteriovenous fistula shunting into two connected venous pouches was diagnosed due to the obvious steal phenomenon in the right VA. Angiography further revealed an $8 \mathrm{~mm}$ berry aneurysm of the right MCA which was surgically clipped, resulting in complete recovery. An endovascular approach was preferred for management of the extracranial arteriovenous shunting lesion.

\section{Endovascular treatment}

Initially, the arterial route was chosen for occlusion of the arteriovenous shunt. The goal was to occlude the fistula by placement of embolic material as close to both fistula points (proximal and distal) as possible. To disconnect the shunt flow from the vertebrobasilar circulation, we first attempted to navigate a detachable balloon from the left VA. This was not possible due to wall irregularities, which caused significant friction to the catheter, and due to low retrograde flow in the right VA.

Therefore we decided to use coils, but even though both VAs were large, advancing the microcatheter to the distal fistula site was still impossible because of friction. Detachable coils (GDC) had to be deployed proximally to the right posterior inferior cerebellar artery (PICA), resulting in subtotal occlusion (figure 2A).

In the same session, a guiding catheter was placed within the right subclavian artery (SA) and a microcatheter was navigated into the short segment of the proximal right vertebral artery caudally supplying the a.v. fistula. Here, tight packing of GDC resulted in total occlusion of the feeding vessel. The final control showed reduction of the shunt flow and occlusion of the smaller venous pouch, but a remaining filling of the second larger pouch via the distal right VA supplied by collateral vessels (ascending, deep cervical, occipital and radicular arteries, figure 2B). Six months later, a follow-up arteriogram still showed an open a.v. shunt (figure 2C). Reaching the fistula site through the deep cervical artery in another session proved impossible because of the tortuousity and size of this branch. Direct puncture or surgical exposure and cannulation of the VA were discussed but not performed because of the risk of creating a new fistula in the highly fragile vascular system.

Therefore, transfemoral venous catheterization of the fistula site was undertaken despite the fact that even close analysis of the late venous phases did not provide any information on the fistula's final venous exit. The dilution of contrast medium injected intra-arterially was too high and the search for a draining vein was performed randomly in the brachiocephalic venous system. Blocking the venous outflow by partial mechanical obstruction with the guiding catheter was helpful in clarifying the venous anatomy (figure $3 \mathrm{~A}$ ). After successful catheterization of the large pouch, we were unable to navigate a microcatheter further into the feeding segment of the distal VA. Angiographically, no other venous exit was recognizable, nor was any connection to the adjacent vertebrospinal venous system revealed.

Therefore, we considered occlusion of the narrowed draining exit of the pouch itself safe. After placement of numerous detachable and fibered coils, the control angiogram showed complete stagnation of the flow in the right VA and no remaining filling of the pouch in the very late phases.

A follow-up after three months showed persistent occlusion of the fistula and a decrease in 


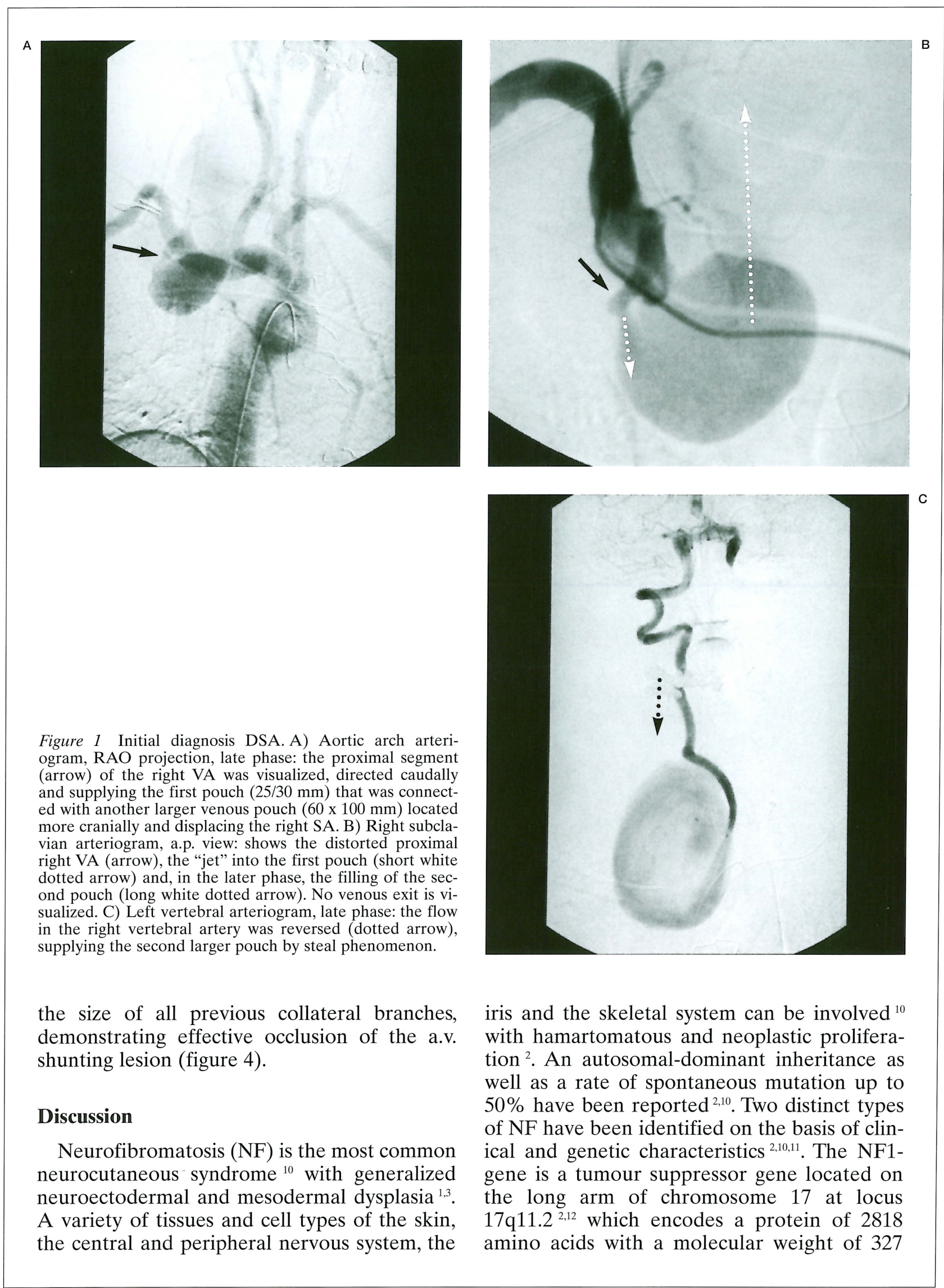




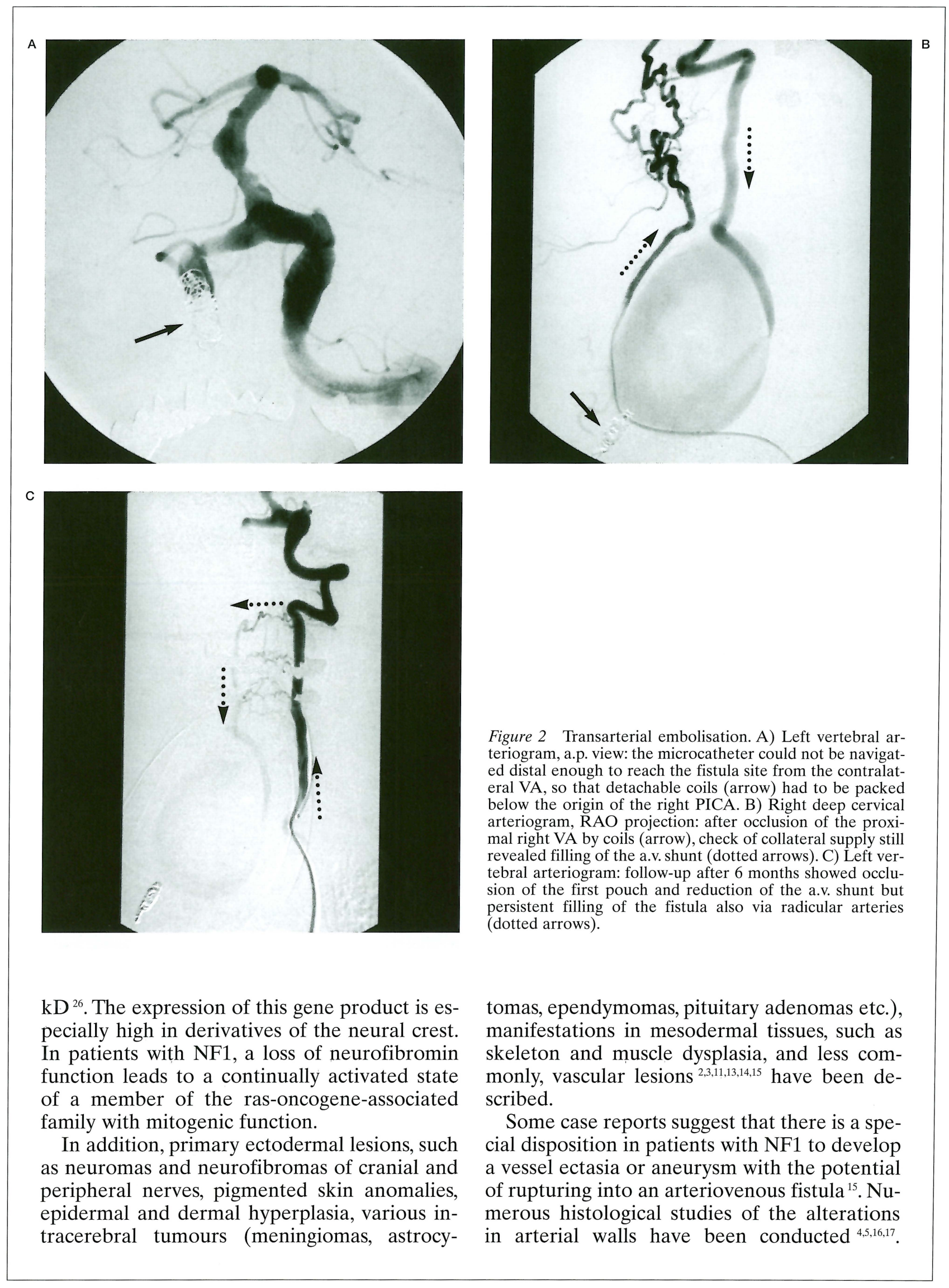


Reubi ${ }^{16}$ has classified three categories according to the diameter of the vessel:

1) The pure intimal form in arteries with a diameter of 0.05 to $0.4 \mathrm{~mm}$ with concentric growth of the intima and thinning of the media.

2) The nodular form with nodular growth of spindle or epithelioid cells in the media or in all wall layers in arteries with a diameter of 0.1 to $0.7 \mathrm{~mm}$.

3) The intimal aneurysmal form in arteries with a diameter of 0.5 to $1 \mathrm{~mm}$ with eccentric fibrous proliferation of the intima, a small number of sparsely distributed spindle cells in the intima and media, smooth muscle fibrosis and elastica fragmentation.

Moreover, Feylter ${ }^{17}$ described an epithelioid form and a more severe, intimal form with eccentric fibrous intimal thickening and small aggregates of spindle cells in the intima. Salyer et $\mathrm{Al}^{4}$ suggested that the foci of spindle and epithelioid proliferation in the vascular walls are of Schwann cell origin which undergo degenerative, e.g. fibrotic and mucinous changes secondarily. In contrast, Greene ${ }^{5}$ found a marked increase in ground substance with thinning and loss of the media, fragmentation to complete disappearance of the elastic lamella, and widely scattered nodular aggregates of smooth muscle cells in small vessels. It has been suggested that these changes cause primary mesodermal dysplasia.

A second type of vascular lesions in the major vessels such as the aorta, the carotid or proximal renal arteries has also been described. In sections surrounded by neurofibromatous or ganglioneuromatous tissue, the authors discovered intimal proliferation, thinning of the media, and fragmentation of elastic tissue leading to stenosis or aneurysmal formation. On the one hand, vascular fragility can more easily be explained by the loss of elastic tissue in the arterial walls. On the other hand, several authors suggest the possibility of a generalized connective tissue abnormality based e.g. on experiences during surgery or on the fact that some NF1 patients exhibit premature facial aging ${ }^{18}$. Complex vascular anomalies of the cerebral circulation are associated with the risk of spontaneous haemorrhage ${ }^{1,2,11,13}$; whether the risk is higher compared to the normal population is not known.

Symptoms related to the fistula described here were bruits and neck pain. In some cases,
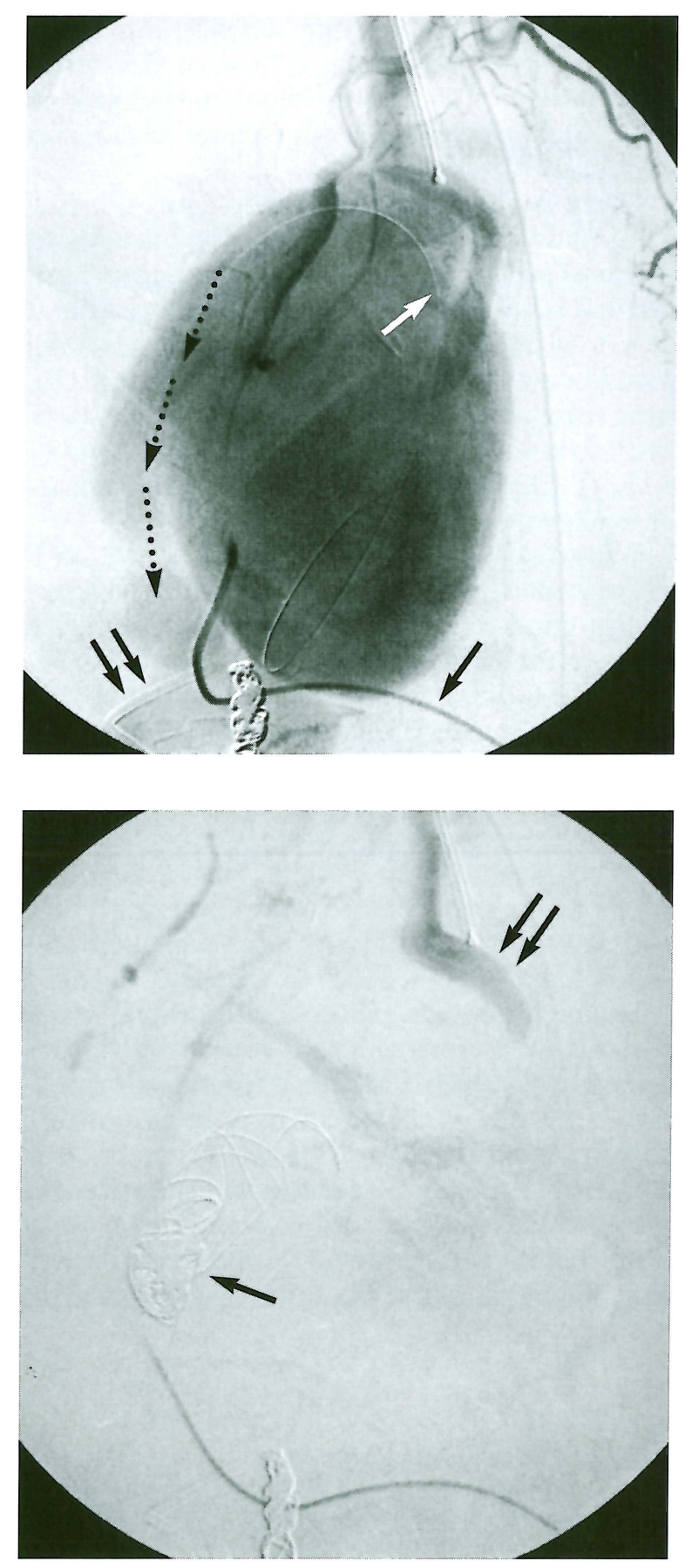

Figure 3 Transvenous embolisation. A) Right deep cervical arteriogram, LAO projection, late phase: retrograde navigation of the guiding catheter (double arrows) into the venous outflow of the pouch (note: not visualized before!); arterial catheter placed in the right deep cervical artery (arrow), and microcatheter (white arrow) navigated into the large venous pouch. B) Same arteriogram post embo: attempts of retrograde navigation into the distal right VA for arterial occlusion of the fistula site failed: placement of detachable and fibered coils (arrow) within the single venous exit finally resulted into complete stagnation of a. v. shunting flow (double arrow). 
neurological symptoms may develop due to intraspinal space occupying ectasia of the vertebral artery $1,2,8,13,14,15,19,20$ with spinal cord compression. Steal phenomena may cause brain and spinal cord dysfunction ${ }^{9}$.

There are only a few published cases of treatment of aneurysms or arteriovenous fistulas of the cerebral arteries associated with neurofibromatosis type $\mathrm{I}^{1,2,8,13,1,1,1,1,19,20}$. Methods of treatment described included proximal ligation, trapping procedures, direct surgical exposure and closure, combined balloon embolisation and surgical ligation and embolisation with balloons or coils ${ }^{9}$. Table 1 summarizes the published results of management in cases similar to ours.

Schievink ${ }^{2}$ was able to occlude a VA-AVF associated with NF type 1 by staged surgery with a good outcome in one of two cases. $\mathrm{He}$ stressed the surgical problems arising from increased vascular fragility and concluded that these lesions should be treated by endovascular means.

From the technical point of view, endovascular management of vertebral arteriovenous fistulas associated with NF1 is similar to other AVFs as described by several authors $9,21,22,23,24,25$ and are treated mainly by occlusion with detachable balloons, leading to excellent results.

Halbach ${ }^{9}$ reported successful treatment of 20 patients with vertebral AVF caused by various diseases, of which 19 were treated endovascularly. Like others ${ }^{24,25}$, Halbach ${ }^{9}$ pointed out that proximal and distal balloon occlusion as close as possible to the fistula site is necessary. $\mathrm{He}$ further discusses the difficulties of placing a distal balloon from the ipsi - and contralateral side. We blocked the steal flow within the right distal VA first, disconnecting the a.v. shunt from the cerebral circulation. Negoro ${ }^{13}$ recently reported a case of a left VA aneurysm that was effectively treated by proximal balloon occlusion. He points out the importance of anastomotic channels that can reconstitute the aneurysm. If those collateral vessels are significantly enlarged, they can sometimes also be used as an approach to the lesion as already described by Moret ${ }^{22}$.

Today's improved endovascular devices help avoid some of the problems and risks in treatment that existed 20 years ago. Nevertheless, in complex arteriovenous lesions, as described here, it might be difficult to close the a.v. shunt via transarterial route only.

In such a situation, the transvenous approach is a valuable alternative, as it is in the treatment of cranial dural arteriovenous fistulas. In fistulas with a sole draining vein and with no connection to the spinal venous system, it can be sufficient to occlude this outflow itself. The given venous anatomy may be difficult to assess by arterial angiography and endovenous navigation of catheters is often more challenging. However, if one succeeds, complete closure of the fistula is achievable safely and effectively.

We also took direct percutaneous puncture of the venous pouch into consideration. In our opinion, regarding the minor clinical symptoms, the advantage of direct access to the fistula did not outweigh the risk of thoracic or mediastinal bleeding by rupturing the fragile venous wall. Direct puncture should be considered a last resort, when every other approach has already failed and a patient presents with progressive clinical symptoms.

Table 1 AVF associated with $\mathbf{N F} \mathbf{1}$

\begin{tabular}{|llllll|}
\hline Source & No.of patients & Localization & Treatment & Result & Outcome \\
\hline Deans (5) & 3 & VA & proximal ligation & ncomplete & improved \\
\hline & & VA & balloons+coils & complete & improved \\
\hline & ECA & coils+resection & complete & improved \\
\hline Schievink (6) & 1 & VA & ligation+resection & complete & new minor deficit \\
\hline Parkinson (12) & 1 & VA & surgical resection & complete & no deficit \\
\hline improved = preexisting neurological deficit improved & & & \\
\hline
\end{tabular}




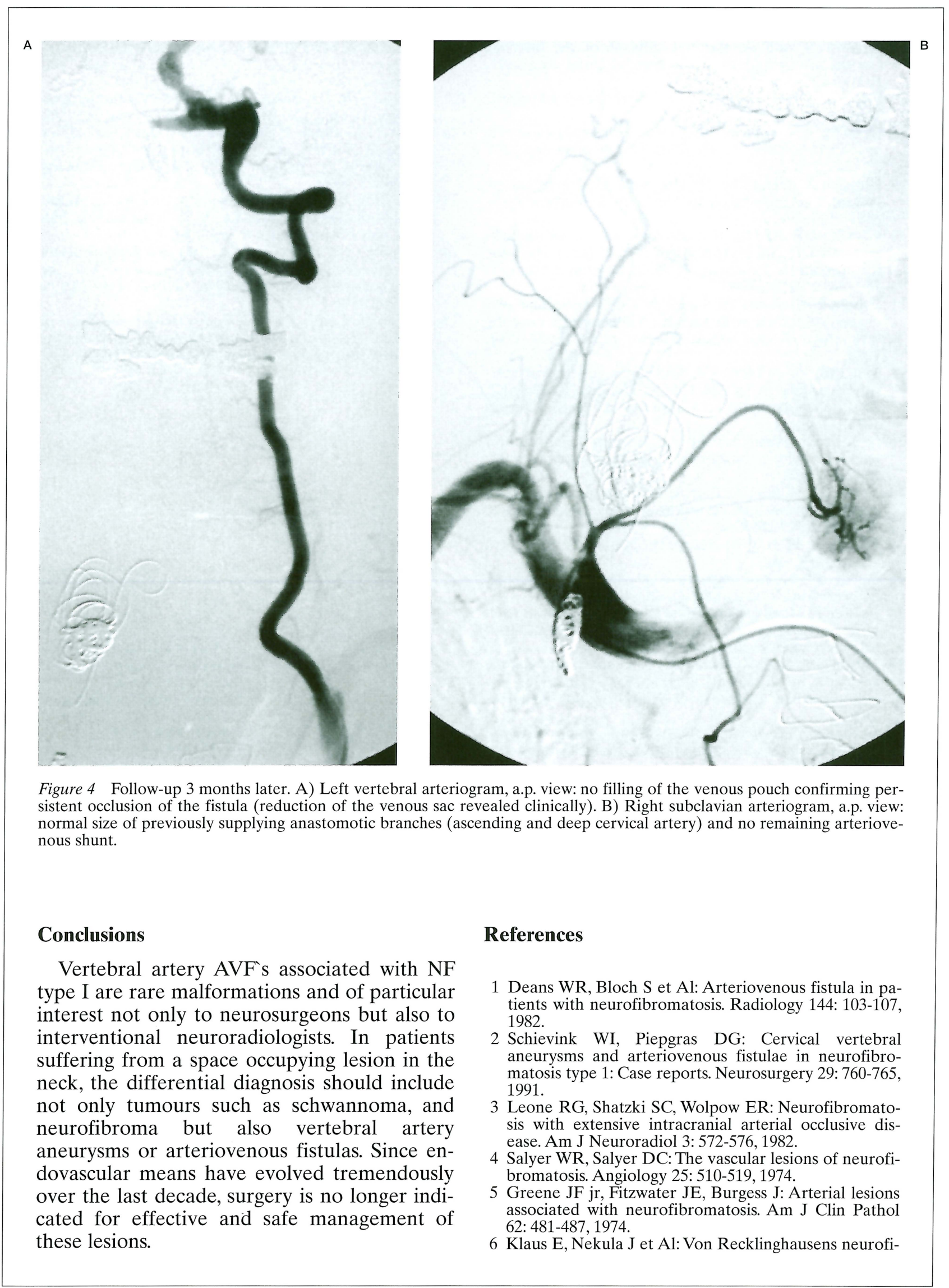


bromatosis with intracranial stenosis of the internal carotid artery and orbitosphenoidal bone dysplasia. ROFO 129: 250-252, 1978.

7 Piepgras U: Neurocutane Systemerkrankungen. Thieme, Stuttgart 1984: 22-54

8 Detwiler K, Godersky JC, Gentry L: Pseudoaneurysm of the extracranial vertebral artery. J Neurosurgery 67 : 935-939, 1987.

9 Halbach V, Higashida R, Hieshima G: Treatment of vertebral arteriovenous fistulas. Am J Neuroradiol 8: 1121-1128, 1987.

10 Wiestler OD, Radner H: Pathology of neurofibromatosis 1 and 2. In: SM Huson, Hughes RAC (eds): The neurofibromatosis. Chapman \& Hall, London 1994: 135159.

11 Sobota E, Ohkuma H, Suzuki S: Cerebrovascular disorders associated with von Recklinghausens neurofibromatosis: A case report. Neurosurgery 22: 544-549, 1988.

12 Marchuk DA, Collins FS: Molecular genetics of neurofibromatosis 1. In: SM Huson, Huighes RAC (eds): The neurofibromatosis. Chapman \& Hall, London 1994: 23-49.

13 Negoro M, Nakaya $\mathrm{T}$ et Al: Extracranial vertebral aneurysm with neurofibromatosis. Neuroradiology 31 : 533-536, 1990.

14 Pentecost M, Stanley P et Al: Aneurysms of the aorta and subclavian and vertebral arteries in neurofibromatosis. Am J Dis Child 135: 475-477, 1981.

15 Parkinson D, Hay R: Neurofibromatosis. Surg Neurol 25: 109-113, 1986.

16 Reubi F: Neurofibromatose et lesions vasculaire. Schweiz Med Wochenschr 75: 463-465, 1945.

17 Feylter F: Über die vaskuläre Neurofibromatose nach Untersuchungen am menschlichen Magen-Darmschlauch. Virchow Arch 317: 221-265, 1949.

18 Huson SM: Neurofibromatosis 1: a clinical and genetic overview. In: SM Huson, Huighes RAC (eds): The neurofibromatosis. Chapman \& Hall, London 1994: 160203.

19 Schubiger O, Yasargil MG: Extracranial vertebral aneurysm with neurofibromatosis. Neuroradiology 15 : 171-173, 1978 .
20 Okhata N, Ikota T et Al: A case of multiple extracranial vertebral artery aneurysms associated with neurofibromatosis. No Shinkei Geka 22: 637-641, 1994.

21 Lasjaunias P, Berenstein A: Surgical Neuroangiography. Vol. II. Springer, Berlin Heidelberg 1987: 212-226.

22 Moret J, Lasjaunias P, Doyon D: Occipital approach for treatment of arteriovenous malformations of the vertebral artery by balloon occlusion. Neuroradiology 17:269-273, 1979.

23 Berenstein A, Kricheff II: Treatment of vascular abnormalities of the vertebral artery with detachable balloons. Am J Neuroradiol 1: 358, 1980.

24 Miller RE, Hieshima GB et Al: Acute traumatic vertebral arteriovenous fistula: Balloonocclusion with the use of a contralateral approach. Neurosurgery 14 (2): 225-229, 1984.

25 Debrun G, Legre J et Al: Endovascular occlusion of vertebral fistulas by detachable balloons with conservation of the vertebral flow. Radiology 130: 141-147, 1979.

Dr Goetz Benndorf

Neuroangiography,

Department Radiology

Virchow Hospital, Charite,

Humboldt University Berlin

Augustenburger PL. 1

13353 Berlin, Germany

e-mail:g.benndorf@charite.de 